\title{
Condición de salud bucal de una población víctima del conflicto armado del municipio de Girardota, Antioquia
}

\section{Oral health condition of a population victim of the armed conflict in the municipality of Girardota, Antioquia}

\section{Estado de saúde bucal de uma população vítima do conflito armado no município de Girardota, Antioquia}

\author{
LL Castrillón-Isaza ${ }^{1}$ \\ M Herrera-Montes ${ }^{2}$ \\ MI Lasso-Álvarez ${ }^{3}$ \\ N Muñoz-Bustamante 4 \\ RY Salcedo-Cueto 5 \\ BD Vásquez-Arroyave ${ }^{6}$ \\ Clara Liliana Gallego-Gómez ${ }^{7}$
}

Recibido: 12 de marzo 2021

Aprobado: 5 de agosto 2021

Publicado: 23 de septiembre de 2021

Cómo citar este artículo:

Castrillón-Isaza LL, Herrera-Montes A, Lasso-Álvarez MI, Muñoz-Bustamante N, Salcedo-Cueto $\mathrm{RY}$, Vásquez-Arroyave $\mathrm{BD}$, et al. Condición de salud bucal de una población víctima del conflicto armado del municipio de Girardota, Antioquia. Revista Nacional de Odontología. (2021);

17(1), 1-17. doi: https://doi.org/10.16925/2357-4607.2021.01.01

Reporte de caso. https://doi.org/10.16925/2357-4607.2021.01.01

Universidad Cooperativa de Colombia, Envigado, Colombia.

ORCID: https://orcid.org/0000-0003-2897-6016

2 Universidad Cooperativa de Colombia, Envigado, Colombia.

ORCID: https://orcid.org/0000-0002-4108-3119

3 Universidad Cooperativa de Colombia, Envigado, Colombia.

ORCID: https://orcid.org/0000-0003-4892-5435

4 Universidad Cooperativa de Colombia, Envigado, Colombia.

ORCID: https://orcid.org/0000-0002-6992-4063

5 Universidad Cooperativa de Colombia, Envigado, Colombia. ORCID: https://orcid.org/0000-0001-7109-1446

6 Universidad Cooperativa de Colombia, Envigado, Colombia. ORCID: https://orcid.org/0000-0001-8107-0964

7 Universidad Cooperativa de Colombia, Envigado, Colombia.

Correo electrónico: clara.gallego@campusucc.edu.co ORCID: https://orcid.org/0000-0001-9317-551X 
2 Condición de salud bucal de una población víctima del conflicto armado del municipio de Girardota, Antioquia

\section{Resumen}

Introducción: la historia del conflicto armado en Colombia ha dejado miles de víctimas que actualmente continúan afrontando graves consecuencias en su calidad de vida, lo que se ve reflejado en su situación de salud y especialmente en su salud bucal.

Objetivo: describir la condición de salud bucal de la población víctima del conflicto armado del municipio de Girardota, Antioquia.

Materiales y métodos: se realizó un estudio cuantitativo descriptivo en una población voluntaria de 52 personas. Por medio de una encuesta estructurada y un examen clínico para el índice de placa de Silness y Löe, índice periodontal básico, COP-M, y de percepción de la situación y necesidad de prótesis.

Resultados: el promedio de edad de 40,5 años, el $75 \%$ eran mujeres, a los estratos 1 y 2 , con un grado de escolaridad de básica primara en el $42 \%$, e ingresos menores a 1 SMMLV en el 60,5\%. El índice COP-D fue de 11 y el COPD-M de 13, la prevalencia de caries fue del $80 \%$, presencia de gingivitis en el $56.9 \%$, y periodontitis del $41,2 \%$, el 55,8 \% presentaron necesidades de prótesis. El 30,8\% asistió a consulta en el último año, en un $40 \%$ por dolor o caries. El $62 \%$ considera regular su estado de salud bucal, y el $87,8 \%$ considera necesitar tratamiento odontológico.

Conclusiones: las estrategias de intervención dirigidas a esta población deben estar enfocadas en la rehabilitación oral para suplir sus necesidades.

Palabras clave: conflicto armado, víctimas, desplazamiento, salud bucal.

\section{Abstract}

Introduction: The history of the armed conflict in Colombia has left thousands of victims and displaced persons who are currently facing serious consequences for their quality of life, which is reflected in their health and especially in their oral health.

Objective: Describe the oral health condition of the population victim of the armed conflict of the municipality of Girardota, Antioquia.

Materials and methods: A descriptive quantitative study was conducted on a sample of 52 people. A structured survey and clinical examination was conducted for the Silness and Löe index, basic periodontal index, COP-M, situation and need for prosthetics.

Results: The average age was 40.5 years, $75 \%$ were women, mostly from 1 and 2 social classification, with a basic elementary schooling grade in $42 \%$ of the participants, and incomes less than minimums salary in $60.5 \%$. The COP-D index was 11 and COPD-M was 13 , the prevalence of cavities was $80 \%$, the presence of gingivitis at $56.9 \%$, and periodontitis of $41.2 \%, 55.8 \%$ had prosthetic needs. $30.8 \%$ attended consultation in the last year, $40 \%$ for pain or decay. $62 \%$ consider poor their oral health status, and $87.8 \%$ consider that they need dental treatment.

Conclusions: We found poor oral health conditions in the population. Population intervention strategies should be focused on oral rehabilitation to attendant their needs.

Keywords: Armed conflict, Victims, Displacement, Oral health, socioeconomics conditions.

\section{Resumo}

Introdução: a história do conflito armado na Colômbia deixou milhares de vítimas que atualmente continuam enfrentando graves consequências em sua qualidade de vida, que se refletem em sua situação de saúde e, principalmente, em sua saúde bucal. 
LL Castrillón-Isaza, M Herrera-Montes, MI Lasso-Álvarez, N Muñoz-Bustamante, RY Salcedo-Cueto, BD Vásquez-Arroyave, Clara Liliana Gallego-Gómez

Objetivo: descrever o estado de saúde bucal da população vítima do conflito armado no município de Girardota, Antioquia.

Materiais e métodos: foi realizado um estudo quantitativo descritivo em uma população voluntária de 52 pessoas. Através de um inquérito estruturado e de um exame clínico para o índice de placa de Silness e Löe, índice periodontal básico, COP-M, e percepção da situação e necessidade de próteses.

Resultados: na média de idade de 40,5 anos, 75\% eram mulheres, nos estratos 1 e 2, com ensino fundamental completo em $42 \%$ e renda inferior a 1 SMMLV em 60,5\%. O índice COP-D foi de 11 e o DPOC-M foi de 13, a prevalência de cárie foi de $80 \%$, a presença de gengivite em $56,9 \%$ e a periodontite foi de $41,2 \%, 55,8 \%$ tinham necessidade de prótese. 30,8\% compareceu a uma consulta no último ano, 40\% por dor ou cárie dentária. 62\% consideram sua saúde bucal regular e $87,8 \%$ consideram que precisam de tratamento odontológico.

Conclusões: as estratégias de intervenção direcionadas a essa população devem ter como foco a reabilitação oral para atender às suas necessidades.

Palavras-chave: conflito armado, vítimas, deslocamento, saúde bucal.

\section{Introducción}

Colombia ha sido un país afectado por un conflicto armado interno, que en su historia reciente, tuvo su inicio en los años cincuenta con la rivalidad entre los partidos políticos Liberal y Conservador (1). Esto derivó en la violencia bipartidista durante varios años y, entre otras causas, da origen a diferentes grupos al margen de la ley, que se suman a la ya difícil situación nacional. Este conflicto se agudiza en los años noventa con el surgimiento de la grupos de extrema derecha, llevando así al país a varias décadas de violencia rural y urbana, desplazamiento forzado, asesinatos, desapariciones y demás crímenes que van en contra de los derechos humanos, sin que la nación pudiera retomar el control y reprimir las acciones de estos grupos.(2)

En el presente el país continúa confrontando secuelas de más de 50 años de conflicto armado interno y violencia, que han derivado en problemas sicosociales, afectación de la infraestructura física, económica, institucional y social del país, que se ha visto reflejada en los problemas de salud mental y en el bienestar de la población (3), especialmente a la población víctima del conflicto.

Se consideran víctimas, según la normativa colombiana, a aquellas personas o grupos que han sufrido daños como consecuencia de infracciones al derecho internacional humanitario (DIH). Así como a las personas que hayan sufrido daño al intervenir para asistir a las víctimas (Ley 1448/2011, de 10 de junio) (4). Situación que se vuelve más crítica cuando las consecuencias individuales y colectivas de la violencia, se unen con las condiciones de vulneración de los derechos humanos, como el derecho las condiciones básicas en salud, educación, equidad, justicia y participación política, los cuales también suelen ser vulnerados en escenarios violentos.(5) 
4 Condición de salud bucal de una población víctima del conflicto armado del municipio de Girardota, Antioquia

El desarrollo de la Ley de víctimas busca a través de política pública del Estado asegurar los derechos a la verdad, la justicia, la reparación y la no repetición estipulados en la ley (5), sin embargo, después de una década de promulgación de la Ley, se siguen observando un aumento la vulneración de la población víctima.

Conforme a lo reportado por la Consultoría para los Derechos Humanos y el Desplazamiento (CODHES), Colombia se catalogaba como el segundo país con el mayor número de personas desplazadas, después de Sudán.(6) Para el año 2015, la CODHES informó que se desplazaron 44.791 personas, de las cuáles Bogotá es la ciudad con mayor recepción con 30.596 personas.(7)

En 2011 se crea el Registro único de Víctimas (RUV), y desde allí se puede tener información en tiempo real sobre el número de víctimas de acuerdo a cada ciudad del país, facilitando el real conteo y aplicación de políticas a esta población (3). Para el año 2020, se tiene un registro de más de nueve millones de víctimas según la unidad del Registro Único de Víctimas (RUV) (8), poblaciones que siguen siendo altamente vulnerables y necesitan atención constante (2).

La población vulnerable se considera como un grupo de personas en estado de desprotección y exclusión, donde las inequidades por diferencias socioeconómicas, culturales y de estilo de vida, en conjunto con el sexo, la raza, la edad y la susceptibilidad genética, amenazan la salud en general e incrementan el riesgo de desarrollar enfermedades orales (9).

Como un mecanismo de enfrentar esta situación y su obligación de proteger los derechos de la población desplazada abarcando su derecho a la salud que, en Colombia, se garantiza a través del Sistema General de Seguridad Social en Salud (SGSSS), y que con la Ley 387 (1997) delegó a las entidades públicas del Sistema la responsabilidad de implementar procesos para mejorar el acceso de la población desplazada a los servicios de asistencia médica, quirúrgica, odontológica, psicológica, hospitalaria y de rehabilitación (10).

El acceso a los servicios de salud se da, a través de la pertenencia a un régimen subsidiado o contributivo, por medio de planes (Plan de Intervenciones Colectivas PIC, Plan de beneficios y Plan de Salud Territorial PST). A pesar de esta legislación y de la existencia del Programa de Atención Psicosocial y Salud Integral a Víctimas (PAPSIVI), la atención a las víctimas del conflicto armado no evidencia una atención integral ni diferencial. Un fenómeno que deteriora la calidad de vida y la salud de esta población, y crea condiciones de vulnerabilidad y desigualdad al momento de acceder a los servicios de salud.(10)

Aunque en los últimos años la población víctima de la violencia en Colombia ha tenido una mayor visualización, lo que ha generado un impacto favorable en algunas 
de sus condiciones de vida, continúa siendo una población con inmensas dificultades en todos los ámbitos. Especialmente en sus condiciones de salud, debido entre otras a los factores sociales, económicos y de inequidad en el acceso a los servicios de salud.

La salud como derecho en la víctima del conflicto armado representa una necesidad vital, se espera que esta nueva etapa del postconflicto responda a las necesidades identificadas ante este derecho. La respuesta social organizada debe partir de un análisis propio de la víctima a sus necesidades en salud, y de diagnósticos que identifiquen las carencias del individuo y en esta misma lógica se construyan respuestas sostenibles, y que superen las visiones mecanicistas de la atención en salud.(3)

Hasta el momento, las investigaciones publicadas sobre la situación de salud en grupos vulnerables son limitadas. Más aún cuando involucran el componente salud bucal; y no especifican condiciones particulares que son necesarias para comprender los procesos sociales que se presentan al interior de grupos específicos.(11)

Teniendo en cuenta la importancia de lo anteriormente expuesto, el presente proyecto buscó describir la condición de salud bucal de la población víctima del conflicto armado del Municipio de Girardota, Antioquia.

\section{Materiales y métodos}

Se realizó un estudio cuantitativo descriptivo. El área de estudio fue la población víctima del conflicto armado del municipio de Girardota, del departamento de Antioquia, registrada en el RUV (Registro Único de Víctimas). Se contó con autorización por parte de la Alcaldía y Secretaria de Gobierno en su dependencia de la Unidad de Atención de Víctimas, la cual proporcionó una base de datos de 100 personas del total de su registro.

Se realizó una convocatoria a las personas en dos ocasiones (área rural y urbana), y se contó con la participación de 57 personas, atendidas por medio de jornadas de salud en la vereda San Andrés y en la cabecera Municipal. Durante la jornada 5 personas decidieron no participar del estudio, quedando una muestra final de 52 personas.

Para la recolección de la información se realizó una encuesta estructurada y un examen clínico. Para la encuesta se tomaron algunas referencias del cuestionario utilizado en el IV Estudio Nacional de Salud Bucal, la encuesta constaba de 51 preguntas, abiertas y cerradas, divididas en cinco ítems: (I) Información General Encuestado, (II) Autopercepción en salud oral, (III) Hábitos de Higiene Oral, (IV) Acceso a Atención 
6 Condición de salud bucal de una población víctima del conflicto armado del municipio de Girardota, Antioquia

Odontológica, (V) Hábitos nocivos. La encuesta se probó y ajustó mediante una prueba piloto y fue aplicada finalmente por una persona entrenada para tal fin.

En el examen clínico se realizó el índice de placa de Silness y Löe modificado e índice periodontal básico (IPB), estado de la dentición mediante COP-M, situación y necesidad de prótesis. Dichos exámenes estuvieron a cargo de un examinador y un auxiliar, calibrados de manera teórica y práctica con el objetivo de obtener registros de calidad.

Se realizó una lectura inicial de los datos, se categorizaron las respuestas de las preguntas abiertas. Posteriormente se realizó un análisis univariado para la descripción de las características sociodemográficas, clínicas y de hábitos de salud oral, mediante la estimación de frecuencias relativas, absolutas y medidas de resumen según la naturaleza de la variable.

El análisis bivariado se realizó para explorar la asociación entre las características sociodemográficas y los hábitos de salud oral con los hallazgos clínicos descritos mediante las pruebas Chi cuadrado de Pearson y $\mathrm{H}$ de Kruskal Wallis, para esta última se realizaron análisis de comparaciones múltiples a través de la prueba $U$ de Mann Whiney para identificar diferencias estadísticamente significativas entre los grupos comparados.

La presentación de la información se realizó mediante tablas y los gráficos de barras y cajas y bigotes; en todos los análisis se tomó un valor P de significación estadística menor a 0,05 realizados mediante el software IBM SPSS ${ }^{\circledR} 25$.

El estudio cumplió con los requerimientos éticos de la investigación en salud, y se clasifica como una investigación de mínimo riesgo. Se contó con la participación voluntaria, garantizando por medio de la firma del consentimiento informado. Esta investigación fue aprobada por el Comité de Bioética de la Universidad Cooperativa de Colombia sede Medellín, Envigado

\section{Resultados}

\section{Características sociodemográficas}

Se encuestaron 52 personas, cuyo promedio de edad fue de 40,5 años (SD $\pm 14,5$ ), con un mínimo de 18 y un máximo de 70 años; la mayoría perteneciente al género femenino (75\%). El 93,6 \% de la población pertenecía a los estratos socioeconómico1 y 2 , procedentes en su mayoría de la zona rural (60,8 \%); un alto porcentaje se encuentran casados/unión libre (52,9 \%) y soltero (35,3\%). En cuanto al nivel educativo se encontró similitud en los niveles de secundaria (48,1 \%) y básica primaria (42,3 \%); la 
mayoría manifestó que actualmente no se encontraba trabajando $(78,4 \%)$ y que sus ingresos mensuales eran menores a 1 SMMLV (60,5\%). Un $62 \%$ se encuentra afiliado al régimen subsidiado, un 32 \% régimen contributivo y 6 \% no cuenta con régimen de afiliación. Referente al hecho victimizante se encontró que el mayor porcentaje en desplazamiento (63,5\%), un 23,1 \% desplazamiento + otro hecho, un 11,5\% no sabe/ no responde y un $1,9 \%$ otro hecho.

\section{Acceso a servicios de salud}

Un 30,8 \% de la población realizó su última visita al odontólogo entre los 6 meses y un año, seguida de un 28,8 \% en los últimos 6 meses, más de 2 años con un 21,2 \%, entre 1 años y dos años 15,4 \% y 3,8 \% los que nunca asiste al odontólogo; el motivo de esta consulta fue de un 42 \% por una revisión/prevención, un 40 \% por dolor/caries, 12 \% por ortodoncia/aparatología; la experiencia percibida en la última visita al odontólogo en mayoría fue agradable (80\%).

\section{Conocimientos de la población en salud oral.}

El 80,7 \% relaciona la salud bucal con no tener caries dental, el 78,8 \% con cuidarse/ limpiarse la boca y un $76,9 \%$ con no tener los dientes dañados. Con relación al motivo de presencia de caries fue relacionado con un 88,4\% mal cepillado/no cepillarse, un $75 \%$ mal uso o no de seda dental y un 65,3 \% con consumo de dulces/alimentos pegajosos y descalcificación dental o debilidad dental.

La opinión frente a la etiología del sangrado e inflamación de las encías, el 84,6 $\%$ por mal cepillado/no cepillarse, el $75 \%$ por cepillarse fuerte y un $71,1 \%$ con debilidad de las encías y mal uso o no uso de seda dental (tabla 1).

Tabla 1. Conocimientos sobre salud oral.

\begin{tabular}{|c|c|}
\hline \multicolumn{2}{|l|}{ Percepción de boca sana } \\
\hline No tener caries & $80,7 \%$ \\
\hline Cuidarse/limpiarse la boca & $78,8 \%$ \\
\hline No tener los dientes dañados & $76,9 \%$ \\
\hline \multicolumn{2}{|l|}{ Etiología de la caries dental } \\
\hline Mal cepillado/no cepillarse & $88,4 \%$ \\
\hline Mal uso o no de seda dental & $75 \%$ \\
\hline Consumo de dulces/alimentos pegajosos y descalcificación dental o debilidad dental & $65,3 \%$ \\
\hline
\end{tabular}


Condición de salud bucal de una población víctima del conflicto armado del municipio de Girardota, Antioquia

(viene)

\begin{tabular}{lc}
\hline \multicolumn{2}{c}{ Etiología de inflamación gingival } \\
\hline Mal cepillado/no cepillarse & $84,6 \%$ \\
\hline Cepillado fuerte & $75 \%$ \\
\hline Debilidad de las encías y mal uso o no de seda dental & $71,1 \%$ \\
\hline
\end{tabular}

Fuente: elaboración propia

Al indagar la percepción que tienen del estado de la boca se encuentra un 61,2 \% regular, un $22,4 \%$ bueno y un $16,3 \%$ malo; un $68,1 \%$ relata que le gustan sus dientes y un $87,8 \%$ tiene necesidad de algún tipo de tratamiento, en el cual un $44,4 \%$ es reconstruir los dientes, 20 \% ortodoncia, 15,6 \% sacar dientes (exodoncia) (tabla 2).

Tabla 2. Autopercepción del estado de salud.

\begin{tabular}{lll}
\hline \multirow{2}{*}{ Estado percibido de Salud Bucal } & \multicolumn{1}{l}{ Bueno } & $22,4 \%$ \\
\cline { 2 - 3 } & Regular & $61,2 \%$ \\
\cline { 2 - 3 } Nalo & $16,3 \%$ \\
\hline & Quitar el dolor & $2,2 \%$ \\
\cline { 2 - 3 } & Reconstruir dientes & $44,4 \%$ \\
\cline { 2 - 3 } & Sacar dientes & $15,6 \%$ \\
\cline { 2 - 3 } & Ortodoncia & $20,0 \%$ \\
\cline { 2 - 3 } & Prótesis & $4,4 \%$ \\
\cline { 2 - 3 } & Limpieza & $6,7 \%$ \\
\cline { 2 - 3 } & Diseño de sonrisa & $6,7 \%$ \\
\hline
\end{tabular}

Fuente: elaboración propia.

\section{Situación de salud bucal}

El índice de COP-D promedio fue de 11 (SD \pm 7 ), con un valor mínimo de 0 y máximo de 26 dientes que se encontraron cariados, obturados o perdidos por caries. El promedio del índice COP-D Modificado fue de 13 (SD ะ6), con un valor mínimo de 1 y máximo de 26 dientes que se encontraban con caries limitada al esmalte, caries cavitacional con compromiso de dentina, obturados o perdidos por caries.

El $80 \%$ de la población presenta dientes con caries, el 83 \% dientes obturados por caries (O), el $52 \%$ dientes perdidos por caries (P) y el $69 \%$ dientes perdidos por otras razones diferentes (figura 1). 


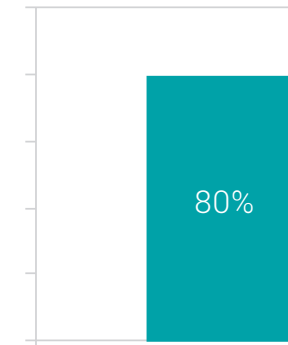

C

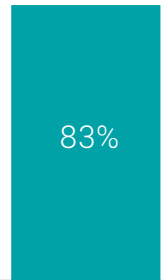

0

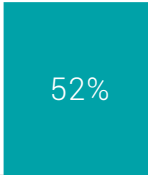

$\mathrm{P}$

Figura 1. Experiencia de caries.

Fuente: elaboración propia.

\section{Índice de placa}

El 76,5\% de la población evaluada presentó un índice de placa deficiente, fue regular en $13,7 \%$, de los evaluados y bueno el $9,8 \%$.

\section{Evaluación periodontal}

En un 56,9 \% de la población evaluada, presentaba gingivitis, un 41,2 \% presentaba periodontitis y el $2 \%$ de la población se encuentra en un estado periodontal sano.

\section{Situación de prótesis.}

El 82,7 \% de la población no presenta ningún tipo de prótesis, un 9,6 \% posee prótesis parcial y un $7,7 \%$ prótesis total.

\section{Necesidad de prótesis.}

El 55.8 \% de la población requiere algún tipo de prótesis, siendo la prótesis parcial el mayor requerimiento evidencia $(40,4 \%)$ y en menor proporción la prótesis total $(15,4 \%)$. 
Tabla 3. Situación de salud bucal.

\begin{tabular}{lll}
\hline \multirow{2}{*}{ Índice de placa } & \multicolumn{1}{l}{ Bueno } & $9,8 \%$ \\
\cline { 2 - 3 } & Regular & $13,7 \%$ \\
\cline { 2 - 3 } Índice periodontal & Deficiente & $76,5 \%$ \\
\hline \multirow{3}{*}{ Situación de prótesis } & Sano & $2,0 \%$ \\
\cline { 2 - 3 } & Gingivitis & $56,9 \%$ \\
\cline { 2 - 3 } & Periodontitis & $41,2 \%$ \\
\hline \multirow{3}{*}{ Necesidad de prótesis } & Ninguna prótesis & $82,7 \%$ \\
\cline { 2 - 3 } & Prótesis parcial & $9,6 \%$ \\
\cline { 2 - 3 } & Prótesis total & $7,7 \%$ \\
\cline { 2 - 3 } & Ninguna prótesis necesaria & $44,2 \%$ \\
\cline { 2 - 3 } & Necesidad de prótesis parcial & $40,4 \%$ \\
\cline { 2 - 3 } & Necesidad de prótesis total & $15,4 \%$ \\
\hline
\end{tabular}

Fuente: elaboración propia.

\section{Discusión}

El pueblo colombiano es víctima del conflicto armado en sus dimensiones sociales, políticas, económicas y de calidad de vida, especialmente en sus condiciones de salud, Esta problemática ha llevado a varios gobiernos a trabajar en pro de la paz de la Nación (3), y al creación de diferentes programas para la atención diferencial de las víctimas del conflicto, sin embargo, continúan las difíciles condiciones de vida para esta población, especialmente en sus condiciones de salud.

El presente estudio encontró como la mayoría (53,2 \%) de los participantes pertenecía al estrato socioeconómico dos, seguido por un 40,4 \% que eran de estrato uno, y un porcentaje más bajo 2,1 \% que hace referencia a estrato tres. Resultados comparables a lo reportado por Agudelo et al. (12) en el que el mayor porcentaje de población desplazada hacían parte del estrato uno y dos. Esto puede indicar algunas de las condiciones y calidad de vida del contexto en donde actualmente habitan; donde se pueden destacar situaciones desfavorables y de carencia en sus viviendas.

Los niveles educativos de los participantes, de la presente investigación, no superan el bachillerato, el 42,3 \% cursó algún grado de escolaridad primaria y el 48,1 \% alcanzó niveles educativos en secundaria. Datos que se pueden comparar con los de Botón Gómez et al. (13) que encontró como el 55 \% de los encuestados apenas ha cursado primaria, el $29 \%$ ha cursado secundaria y alrededor del $14 \%$ no tiene ningún tipo de estudio, situación que dificulta la inserción laboral de ésta población 
y disminuye sus posibilidades de ingresos, como lo reporta Mogollón Pérez et al. (14) quienes describen en su estudio que los bajos niveles educativos son causales de la poca generación de ingresos, perpetuando las condiciones desfavorables de la población.

De acuerdo con el estado civil, Agudelo et al. (12) reportaron como la mayor parte de la población (68,9 \%) convive con su pareja, ya sea casado o en unión libre, resultado comparable con el presente estudio, en el que se encontró que un 52,9\% de la población convivía con su pareja.

Dentro de los resultados se observó que el 25 \% eran hombres y el 75 \% complementario correspondían a mujeres, resultados similares se encontraron en los estudios de Revelo et al.(15), los cuales evidenciaron que el número mayor de víctimas participantes en su estudio fueron del sexo femenino, de igual manera, Castañeda et al. (3) reportaron como el 72,1 \% de la población víctima del conflicto armado participante fueron mujeres, evidenciando la feminización de las víctimas del conflicto; como lo refiere Tortosa "feminización de la pobreza" dado a que las mujeres están cada vez más representadas entre los pobres del mundo. Lo que evidencia como la mujer siempre ha estado en condiciones desfavorables, respecto al hombre, en aspectos que van directamente relacionados a sus oportunidades laborales, ingresos económicos y a su estado de salud.

Lo que en muchos casos conlleva a que las mujeres convivan en contextos de violencias de género, familiar y social que evidencian una desigualdad, situaciones referidas por Alcaraz et al. (6), también este autor refiere como en muchos casos por violencia o abandona, las mujeres se convierten en cabeza de hogar.

Situación que había sido referida previamente por Mogollón Pérez et al. (16) quienes afirman que la prevalencia del género femenino en la cabeza de hogar en esta población son causales de la poca generación de ingresos y la inestabilidad o falta de acceso al sistema laboral, lo que conlleva a que las mujeres terminen siendo el género con mayor afectación respecto a lo económico y laboral. Se ven obligadas a realizar trabajos domésticos e informales, y esto determina un efecto de estancamiento en sus aspectos personales, psicológicos y sociales. Afirmación que se complementa con lo expuesto por Alcaraz et al. (6) quienes consideran que a las mujeres en el momento del desplazamiento les ocurre una alteración en su salud y bienestar, sin embargo, la prioridad para las mujeres no es consultar por su salud, la prioridad es huir con su familia del agresor, que sumado a la escasa preparación académica no les permite incursionar en el mercado asalariado y por ende la oferta se limita a servicios domésticos, servicios sexuales y la mendicidad, a lo que dichos autores mencionan como Mujer vulnerable para trabajo sexual, el trabajo sexual para supervivencia. 
El estudio realizado por Agudelo A et al. (12) muestra que el 63,3 \% de sus encuestados están desempleados y quienes trabajan, lo hacen en empleos informales (72,8\%), oficios domésticos o agricultura, comparable con hallazgos de la presente investigación, en donde el 60,5 \% de desplazados, presentan ingresos inferiores al salario mínimo y el restante solo devenga entre uno o máximo dos, además, del total de los entrevistados, el 78 \% no trabaja formalmente, una causal evidente que pone la atención en salud en una escala baja dentro de sus prioridades al momento de hacer sus gastos de manutención.

Según la red ORMET (17) (el Observatorio Regional del Mercado de Trabajo) la población nacional tiene una tasa de desempleo del 10,2 \%, mientras que para la población desplazada es del 35,5 \%, lo que conlleva a una condición de pobreza crónica cada vez más difícil de superar.

Situaciones que vulneran los derechos humanos en esta población víctima, específicamente en su situación laboral, lo que conlleva al empeoramiento de su calidad de vida, y sus relaciones familiares y sociales, produciendo fuertemente uno de los principales retos para el gobierno para la construcción de la paz en Colombia.

Dentro del tipo de afiliación al régimen de salud se encontró que el 62 \% hace parte del régimen subsidiado. Esto se relaciona con los resultados de Mogollón et al. (16), en donde se menciona que solo un 45 \% de la población desplazada está afiliada de manera contributiva al SGSSS ya que muchos de ellos presentan diversas barreras que obstaculizan el acceso a los servicios de salud de esta manera, debido a que estos grupos cuentan con escasos recursos económicos, debido en gran medida a la pérdida de sus bienes de subsistencia, a los problemas para la generación de nuevos ingresos y al desempleo.

Esta situación, como lo relata Alcaraz et al. (6), hace que las personas deban realizar un camino largo para una consulta en salud y que según Mogollón et al. (6) hace que la población se sienta excluida y marginalizada por parte de los prestadores de servicios de salud, afectando su salud debido a la alta demanda en los servicios y un bajo presupuesto institucional, por lo que Alcaraz et al. afirman que, a pesar de las buenas intenciones por parte de las instituciones éstas no se encuentran preparadas para la atención de la población víctima.

Según Mogollón et al. (16), la suma de lo anterior con la inexistencia de claridad en la definición de los criterios de selección de los beneficiarios de la atención genera exclusión a un amplio número de personas desplazadas.

Esto se relaciona a lo dicho por López et al. (10), quienes afirman que en el financiamiento de los servicios de salud para la población víctima del conflicto armado, existen dificultades como el desequilibrio entre recursos y necesidades, disponibilidad 
de fondos provenientes de fuentes de financiamiento del sistema de salud, administradores y organismos de seguimiento y control. Ello debido al aumento de la vulnerabilidad del derecho a los servicios de salud, dado por la corrupción, la fuerza política y económica de quienes administran. Esta información se considera de importancia para identificar las necesidades monetarias y de acceso a los servicios de salud que sufren la mayoría de las personas desplazadas.

En esta misma medida, las consecuencias económicas son la base responsable para subestimar la salud general y específicamente la salud bucal en el caso de esta población de estudio. Es así como a lo largo de esta indagación dan respuestas en donde excusan que su mal estado de salud va directamente relacionado al poco ingreso monetario, y que por su situación precaria en cuanto a necesidades básicas deben priorizar gastos para el sustento diario en alimentación y techo, de ellos y de las personas con quienes conviven.

Lo cual se puede confirmar según investigación de Agudelo et al. (11) en la que manifiestan que el concepto de la salud está relacionado con el desarrollo familiar, su estado de salud física determina la posibilidad de buscar día a día un sostenimiento para suplir sus necesidades básicas, obtener trabajo, seguir con sus estudios. Lo importante no es mantener la salud diariamente, es controlar la enfermedad cuando está presente con los conocimientos que adquirieron de personas cercanas.

Se vincula el aseo con el significado de salud, con la ausencia de enfermedad, esto a partir de las costumbres. Por otro lado, se identifican los factores de riesgo para enfermedades tanto del contexto de la salud bucal como de la salud en general. El significado en si no es cambiado por el desplazamiento. (11)

También se ha demostrado que existen otras barreras para el acceso a consultas odontológicas diferentes a la condición económica, como lo mencionó Mogollón (16) quien identificó "dificultades relacionadas con el bajo nivel de información, la necesidad de reconocimiento de la condición de desplazado, la integración social en su nuevo entorno inmediato, problemas de salud de diversa índole y su actitud frente a su situación". Resultados también relacionados con los de Revelo et al. (15) dónde el bajo nivel educativo y la falta de recursos fueron las causas más comunes para el origen de los problemas de salud en la cavidad oral.

Si bien la mayor parte de la población asiste citas odontológicas, el motivo más frecuente es consulta preventiva, lo que no responde a la necesidad primordial respecto a salud bucal, pues como lo evidencia la encuesta de la presente investigación, la necesidad primordial se manifiesta en el requerimiento de prótesis parcial y total, detalle que se expone más adelante. Las prestaciones en salud cumplen con la atención preventiva que ellos demandan, pero no llegan a cubrir su necesidad más 
alta que es el requerimiento de prótesis, ya que no están incluidas dentro del Plan de Beneficios y además sus dificultades económicas les impide a acceder a este tipo de atenciones de manera particular.

Además en la población según Agudelo et al. (11) reconocen la boca como una estructura vital para las funciones básicas y se vincula la higiene bucal en las prácticas cotidianas de higiene personal, que se transmite por los adultos significativos, pese a las dificultades propias. Tratan a su manera de asumir los retos saludables que se presenten, con autocuidados aprendidos desde la infancia, que se corresponden a su difícil situación pero que no los desvincula de sus representaciones de bienestar y la importancia de la salud bucal es prioritaria por la existencia de un dolor insoportable, no por las consecuencias que esto trae a nivel general del componente bucal. Perciben que la atención adecuada de los pacientes por parte del profesional evitará su deterioro. (11)

En este estudio, el promedio total del COP-D modificado fue 13 (SD \pm 6$)$, resultados comparables con lo expuesto por Agudelo et al. (12), que describe que el COP Modificado total de las poblaciones desplazadas y destechadas fue de 10,4 entre todos los códigos que hacen parte del mismo. Lo anterior demuestra, que toda la población evaluada ha tenido una experiencia significativa con respecto a la enfermedad y la prevalencia de caries. Tomando como referencia el ENSAB IV(18), se encuentra como $91,58 \%$ ha tenido alguna experiencia con la caries dental y donde también se afirma que hay mayor prevalencia de caries en personas que hacen parte de régimen diferente al subsidiado de afiliación al Sistema de Seguridad Social en Salud (94,35\%). igualmente se evidenció en el estudio ya mencionado de Andrés et al. (12), donde se demostró que el 89,7 \% de las personas tienen historia de caries dental.

Según Agudelo et al. (11) indican que los estudios demuestran que los programas de educación en salud bucal realizados a través de charlas y conferencias motivacionales, cartillas y ayudas audiovisuales, entre otras, pueden ayudar a reducir estos índices de caries gracias a la interacción de las familias.

Durante el examen intraoral que se le realizó a la población, también se encontró que el 9,6 \% cuenta con prótesis parcial, y el 7,7 \% presenta prótesis total, resultados comparables con lo expuesto por Agudelo et al. (12) en su estudio, quienes manifiestan que el 10,3 \% de las personas presentan prótesis parcial removible superior, el 12 \% tienen prótesis total superior y en el maxilar inferior el 3,4 \% presenta prótesis removible y el 1,7 \% prótesis total. Lo que presenta un bajo uso de prótesis en esta población, teniendo como referencia a los datos de la población general reportados en el IV Estudio Nacional de Salud Bucal (18) que reportan una frecuencia del uso de algún tipo de prótesis en un 31,62 \%. 
En cuanto a la necesidad de prótesis se encontró que el 55,8 \% de la población requiere algún tipo de prótesis, resultados mucho mayores teniendo como referencia lo reportado por Agudelo et al. (12) quienes encontraron como entre el 10,5 \% y el 32,8 $\%$, requiere algún tipo de prótesis o cambio de las mismas.

\section{Conclusiones}

A pesar de que los participantes presentan conocimientos básicos en salud bucal, esto no se relaciona con sus condiciones orales, lo que se debe tener en cuenta para el desarrollo de futuros proyectos en las que se consideren campañas de promoción y prevención de salud bucal, para que efectivamente puedan impactar en esta población y que a largo plazo logre el mejoramiento de las condiciones de vida y su desarrollo en un entorno social favorable.

Se establece la necesidad de investigaciones sobre la aplicación de políticas, normatividad y proyectos relacionados con acceso a servicios de salud en la población víctima del conflicto armado en Colombia, en especial a raíz del proceso de paz y la restitución de derechos en el marco de posconflicto.

\section{Conflicto de interés}

Los autores declaran no tener ningún conflicto de interés.

\section{Referencias}

1. Rojas Y, Diaz S. Education and Sensitization on Violence: Seeking to Understand the Victims of the Colombian Armed Conflict. Procedia Soc Behav Sci. 2017;237:562-567.

2. Ramirez L, Veloza M, López A. Acceso a Los Servicios De Salud De Las Víctimas Del Conflicto Armado, En El Escenario Del Postconflicto. Mov Científico. 2016; 10(1):88-94.

3. Castañeda J, Camargo J, López W. Calidad de vida relacionada con la salud en población víctima del conflicto armado en Colombia. Psicol desde el Caribe. 2019; 36(2):132-148.

4. Colombia. Congreso. Ley 1148 de 2011. Congreso de Colombia. 2011 Disponible en: https:// www.unidadvictimas.gov.co/es/ley-1448-de-2011/13653\#: :text=Reseña\%3A, y se dictan otras disposiciones. [Consultado 30 Jun 2020]. 
Condición de salud bucal de una población víctima del conflicto armado del municipio de Girardota, Antioquia

5. Cerón P. El derecho a la participación de las victimas del conflicto armado desde el contexto de la Mesa Municipal de Santiago de Cali en el marco de la Ley 1448 of 2011. Pecedente. 2017; 11:101-136.

6. Alcaraz G, Ochoa S, López Z. Atención en salud a mujeres en situación de desplazamiento: Una mirada desde los prestadores de servicios sociales y de salud. Index Enferm. 2012; 21(3):155-165.

7. Granados H, Loaiza P. El rumbo de la población desplazada en Bogotá-Colombia. Estudio de caso en la localidad de Kennedy 1980-2016. Rev Geográfica Digit. 2017; 28:1-15.

8. Gobierno de Colombia. Unidad para la atención y reparación integral de victimas. Registro único de víctimas. Disponible en: https://www.unidadvictimas.gov.co/es/ruv/37385 [Consultado 11 Jun 2020].

9. Benavides J, Cerón X. Salud oral en poblaciones vulnerables. Rev Criterios. 2017; 24(1): 381-394.

10. López A, Ramírez L, Veloza M, Jiménez C. Financiamiento y calidad de los servicios de salud para la población víctima de conflicto armado en Colombia. Rev Fac Ciencias la Salud Univ del Cauca. 2017; 19(1):34-39.

11. Agudelo A, Zapata D, Martinez E, Gómez L, Camargo D, Vanegas J, et al. La dinámica familiary el fomento de la salud bucal en poblaciones desplazadas y destechadas. CES Odontol. 2007; $20(2): 23-30$

12. Agudelo A, Isaza L, Bustamante D, Martínez C, Martínez C. Perfil epidemiológico bucal en un asentamiento de población desplazada y destechada (Antioquia-Colombia). CES odontolgia. 2008; 21(2):17-24.

13. Botón S, Botero M, Rincón J. El caso del desplazamiento forzado en Colombia: un análisis municipal a partir de regresiones cuantílicas. Equidad y Desarrollo. 2013; (19):77-96.

14. Mogollón A, Vázquez M, García M. Necesidades en salud de la población desplazada por conflicto armado en Bogotá. Rev Esp Salud Publica. 2003; 77(2):257-266.

15. Revelo I, Gutierrez D, Castro C, Rodriguez L. Salud bucodental para comunidades vulnerables y desplazadas de Popayán que viven en condiciones de pobreza. Rev Cuba Salud Pública. 2019; 45(3):1-13. 


\section{LL Castrillón-Isaza, M Herrera-Montes, MI Lasso-Álvarez, N Muñoz-Bustamante,}

RY Salcedo-Cueto, BD Vásquez-Arroyave, Clara Liliana Gallego-Gómez

16. Mogollón A, Vásquez M. Características de la población desplazada que inciden en el acceso a los servicios de salud. Rev Gerenc y Políticas Salud. 2006; 5(11):96-110.

17. Programa Naciones Unidas para el Desarrollo. Ormet. Población desplazada y mercado de trabajo. Disponible en: http://www.co.undp.org/content/colombia/es/home/presscenter/ articles/2014/02/13/ormet-poblaci-n-desplazada-y-mercado-de-trabajo.html [Consultado 13 Feb 2014].

18. Colombia. Ministerio de Salud y Protección Social, MINSALUD. IV. Estudio Nacional De Salud Bucal - ENSAB IV. Bogotá: Minsalud; 2014. 